\title{
¿CÓMO ENSEÑAR CIENCIAS? PRINCIPALES TENDENCIAS Y PROPUESTAS
}

\author{
CAMPANARIO, JUAN MIGUEL y MOYA, AIDA \\ Grupo de Investigación en Aprendizaje de las Ciencias. Departamento de Física. \\ Universidad de Alcalá de Henares. 28871 Alcalá de Henares. Madrid
}

\section{SUMMARY}

In this article we review the main current tendencies and approaches for teaching science. We analyze each one of these proposals and evaluate their main advantages and shortcomings.

\section{INTRODUCCIÓN: LA ENSEÑANZA TRA- DICIONAL Y LA NECESIDAD DE NUEVOS ENFOQUES}

La investigación en didáctica de las ciencias ha identificado diversas dificultades en los procesos de aprendizaje de las ciencias que podríamos denominar «clásicas». Entre estas dificultades cabe citar la estructura lógica de los contenidos conceptuales, el nivel de exigencia formal de los mismos y la influencia de los conocimientos previos y preconcepciones del alumno. En los últimos años se detecta un cierto desplazamiento en los centros de interés de la investigación y se presta cada vez más atención a factores tales como las concepciones epistemológicas de los alumnos, sus estrategias de razonamiento o a la metacognición.

Las concepciones epistemológicas se refieren a las ideas acerca del conocimiento en general o, en nuestro caso, acerca del conocimiento científico: cómo se estructura, cómo evoluciona y cómo se produce (Hammer, 1994). Las concepciones epistemológicas sobre la ciencia guardan relación con las concepciones sobre cómo se aprende el conocimiento científico. Por ejemplo, muchos alumnos piensan que el conocimiento científico se articula en forma de ecuaciones y definiciones que tienen que ser memorizadas más que comprendidas. Hoy sabemos que este tipo de factores constituye un obstáculo formidable para el aprendizaje de las ciencias y es responsable de muchos de los fracasos que registran los enfoques que se proponen para la enseñanza de las ciencias (Linder, 1993). Lo peor de todo es que los factores anteriores no son meros obstáculos pasivos que hay que eliminar, sino verdaderos elementos opositores activos que sesgan y filtran los conocimientos académicos.

Por otra parte, existe amplia evidencia de que, cuando los alumnos abordan el análisis de problemas científicos, utilizan estrategias de razonamiento y metodologías superficiales (Carrascosa y Gil, 1985) o aplican heurísticos importados del contexto cotidiano pero de dudosa utilidad cuando se trabaja con contenidos científicos (Pozo, Sanz, Gómez y Limón, 1991). 
Para acabar de complicar las cosas, en muchas ocasiones las estrategias metacognitivas de los alumnos son realmente pobres. Uno de los «nuevos» problemas detectados en los alumnos de ciencias es que aplican criterios de comprensión limitados, de manera que no siempre son capaces de formular sus dificultades como problemas de comprensión; es decir, no saben que no saben (Otero y Campanario, 1990; Campanario, 1995). Las destrezas metacognitivas son especialmente relevantes en el aprendizaje de las ciencias, dado que la interferencia de las ideas previas obliga a disponer de un repertorio de estrategias de control de la comprensión adecuado que permita detectar fallos en el estado actual de comprensión (Otero, 1990). Como indica Baker, si los alumnos no son conscientes de que mantienen concepciones erróneas sobre los contenidos científicos, es difícil que tomen alguna postura para clarificar su comprensión (Baker, 1991).

A la vista de los problemas anteriores podía parecer que existiese una especie de conspiración cognitiva contra el trabajo del profesor (Pozo, 1987, p. 83). Ante esta realidad anterior parece claro que las estrategias tradicionales de enseñanza de las ciencias son poco eficaces para promover el aprendizaje significativo. Es innegable que en muchas de las aulas predomina un modelo de enseñanza por transmisión. Según Calatayud, Gil y Gimeno, este modelo tiene su fundamento en unas suposiciones inadecuadas (Calatayud, Gil y Gimeno, 1992):

a) Enseñar es una tarea fácil y no requiere una especial preparación.

b) El proceso de enseñanza-aprendizaje se reduce a una simple transmisión y recepción de conocimientos elaborados.

c) El fracaso de muchos alumnos se debe a sus propias deficiencias: falta de nivel, falta de capacidad, etc.

Cómo enseñar más eficazmente es un problema abierto. Por tanto, es conveniente abandonar la noción de método de enseñanza y cambiarla por estrategia de enseñanza. Estas estrategias de enseñanza se concretan en unas actividades de enseñanza en las que «se maneja cierta información procedente de unas determinadas fuentes, mediante procedimientos concretos (asociados a unos medios didácticos) y en relación con unas metas explícitas o implícitas» (García y Cañal, 1995, p. 7). El modo de orientar las estrategias de enseñanza de las ciencias ha sido el objeto central de un apasionado debate y de no pocos trabajos de investigación. Aunque todavía no hemos conseguido una respuesta definitiva al problema de cómo enseñar ciencias, disponemos de criterios más exigentes para analizar y evaluar críticamente las distintas propuestas.

Como punto de partida, los enfoques alternativos a la enseñanza tradicional de las ciencias descartan el modelo del aprendizaje por transmisión hoy unánimemente combatido por los especialistas e investigadores en enseñanza de las ciencias. Una vez descartados enfoques de enseñanza basados únicamente en la transmisión de información, la organización de las actividades de enseñanza que conducen al aprendizaje significativo está lejos de ser evidente o unívoca (Driver, 1988). Precisamente, el objetivo fundamental de este artículo es revisar y analizar críticamente los enfoques más influyentes que se han propuesto para intentar vencer con mayor o menor éxito los muy diversos elementos que configuran las dificultades del proceso de aprendizaje de las ciencias.

En las secciones siguientes se revisan y analizan críticamente algunos de los puntos de vista alternativos que más influencia han tenido y tienen en el área de enseñanza de las ciencias. Los enfoques que se analizan están influenciados por suposiciones y teorías acerca de cómo se aprende. Sin embargo, las teorías del aprendizaje tienden a ser descriptivas, mientras que las teorías de la instrucción tienden a ser prescriptivas. En las teorías sobre el aprendizaje pueden tenerse en cuenta las condiciones en que se desarrolla el proceso de enseñanza. Las teorías sobre la enseñanza deberían tener en cuenta dichas condiciones. Pero, además, las teorías sobre la enseñanza de las ciencias deben tener en cuenta factores tales como lo que el alumno ya sabe, la especial naturaleza de las disciplinas científicas, la organización social de la enseñanza, las características sociales y cognitivas de los alumnos, sus concepciones epistemológicas y destrezas metacognitivas, las relaciones psicosociales en el aula, los factores motivacionales, los recursos y medios disponibles, etc. No todos los enfoques que se revisan en este artículo tienen en cuenta todos los factores anteriores de manera explícita. El objetivo que se persigue es disponer de criterios para analizar críticamente las propuestas y elegir, en la medida de lo posible, los aspectos positivos entre las que se consideren más prometedoras.

\section{¿TIENE VIGENCIA EL APRENDIZAJE POR DESCUBRIMIENTO?}

Se tiende a asociar el aprendizaje por descubrimiento a los niveles de enseñanza primaria y secundaria y, de hecho, fue una de las primeras alternativas que se ofrecieron a la enseñanza repetitiva tradicional en estos niveles. Los defensores del aprendizaje por descubrimiento fundamentaban su propuesta en la teoría de Piaget. Esta teoría alcanzó gran difusión en un momento en que muchos profesores, especialmente de ciencias, buscaban alternativas al aprendizaje memorístico y repetitivo y al fracaso generalizado en la enseñanza tradicional. Tras años de dominación del enfoque del aprendizaje receptivo de contenidos, las concepciones piagetanas conducían al aprendizaje por descubrimiento en lo que Novak llamó «un matrimonio de conveniencia» (Novak, 1982, p. 110). La predilección de Piaget por el aprendizaje por descubrimiento se pone de manifiesto en su conocida afirmación según la cual, «cada vez que se enseña prematuramente a un niño algo que hubiera podido descubrir solo, se le impide inventarlo y, en consecuencia, entenderlo completamente» (citado en Pozo y Carretero, 1987, p. 38). 
El aprendizaje por descubrimiento, con su énfasis en la participación activa de los alumnos y en el aprendizaje y aplicación de los procesos de la ciencia, se postulaba como una alternativa a los métodos pasivos basados en la memorización y en la rutina. Dado que este punto de vista prescribe una dirección de enseñanza y aboga por determinadas estrategias, es razonable considerarlo también como una teoría de la enseñanza. Cabe preguntarse si el aprendizaje por descubrimiento tiene algo que aportar hoy día a la enseñanza en los niveles obligatorios e incluso en la universidad. Para ello es conveniente revisar brevemente la experiencia y los resultados del aprendizaje por descubrimiento.

El enfoque de la enseñanza por descubrimiento conoció un gran desarrollo durante los años sesenta y parte de los setenta. Diversos proyectos de renovación educativa siguieron este enfoque en el que se fomenta a toda costa la actividad autónoma de los alumnos. Incluso a veces se llega a rechazar, como señalan Ausubel, Novak y Hanesian, cualquier tipo de guía o dirección del aprendizaje (Ausubel, Novak y Hanesian, 1983). En consonancia con los postulados piagetanos, en el aprendizaje por descubrimiento se presta escasa atención a los contenidos concretos que el alumno debe aprender frente a los métodos (Gil, 1994). Lo importante es aplicar a toda costa las estrategias de pensamiento formal. De acuerdo con este enfoque, la enseñanza debería basarse en el planteamiento y resolución de situaciones abiertas en las que el alumno pueda construir los principios y leyes científicos. Éste sería el método ideal para fomentar la adquisición de destrezas de pensamiento formal que, a su vez, permitirían al alumno resolver casi cualquier tipo de problema en prácticamente cualquier dominio del conocimiento. Además, encontrando sus propias soluciones a los problemas, los alumnos serían capaces de aprender las cosas haciéndolas y ello haría más probable que las recordaran (Pozo y Carretero, 1987). Por otra parte, se argumentaba que la implicación activa en el aprendizaje y el contacto directo con la realidad redundarían en una mayor motivación de los alumnos.

Sin embargo, tanto las evidencias experimentales como los análisis críticos pusieron de manifiesto inconsistencias y deficiencias en el aprendizaje por descubrimiento (Gil, 1994; Pozo y Carretero, 1987; Driver, 1988). Como señala Gil, es muy probable que una búsqueda a tientas por parte del alumno «dé como resultado el aprendizaje de un conjunto de adquisiciones dispersas» (Gil, 1983, p. 30). Otros críticos señalan que en muchas ocasiones la participación activa se confunde con la mera manipulación. No es raro que los alumnos de enseñanza secundaria e incluso de universidad apliquen estrategias de pensamiento nada formales e incluso heurísticas sesgadas por lo que a veces «descubren» otras cosas distintas a las que se pretendía (Rowell y Dawson, 1983). Además, es frecuente que la experiencia empírica refuerza ideas previas erróneas de los alumnos sobre los fenómenos científicos (Gunstone y White, 1981; Driver, 1988). Parece claro, por otra parte, que los alumnos suelen tener dificultades en una de las tareas básicas del aprendizaje por descubrimiento, como es la capacidad para contrastar hipótesis. Diversas investigaciones señalan que la capacidad para eliminar hipótesis mediante la falsación se desarrolla a una edad relativamente tardía (entre los 14 y los 16 años) y no siempre en todos los alumnos (Carretero, 1987).

Otra crítica que se formula al aprendizaje por descubrimiento es que está basado en unas concepciones epistemológicas hoy día superadas. Con su énfasis en la observación y en la formulación de hipótesis, este enfoque tiene mucho que ver con concepciones excesivamente inductivistas sobre la ciencia y el trabajo científico. Lo que empezó siendo una justificación psicológica del aprendizaje se acabó basando en una justificación epistemológica sobre la estructura de la ciencia y de los procesos científicos según concepciones que prestan demasiada atención al proceso de observación y formulación de hipótesis. De hecho, según Hodson, una de las características del aprendizaje por descubrimiento que más facilitó su extensión es que la visión de la ciencia que lo sustenta es más «sencilla» que la de otros modelos de la ciencia y los alumnos pueden comprenderla con más facilidad (Hodson, 1994). Las preferencias por los procedimientos frente a los contenidos es discutible además por otras razones: tal como demuestran las investigaciones sobre la influencia de las ideas previas de los alumnos de ciencias, y en contra de la supuesta independencia del pensamiento formal, los contenidos concretos sí son importantes a la hora de aprender ciencias.

Una de las críticas más certeras al aprendizaje por descubrimiento es la que realiza Ausubel cuando distingue entre aprendizaje memorístico y aprendizaje significativo (Ausubel, Novak y Hanesian, 1983). Según Ausubel, ni todo el aprendizaje receptivo es forzosamente memorístico, ni todo el aprendizaje por descubrimiento es necesariamente significativo. Lo importante no es que el aprendizaje sea receptivo o sea por descubrimiento, sino que sea memorístico o sea significativo. Estas categorías formarían unos ejes independientes («ortogonales» en palabras de Ausubel) que permitirían clasificar las situaciones de aprendizaje en el aula de acuerdo con los componentes según cada uno de los ejes. Así, por ejemplo, la búsqueda de soluciones a problemas complejos por ensayo y error sería un ejemplo de aprendizaje por descubrimiento que difícilmente daría lugar a un aprendizaje significativo.

Las teorías de aprendizaje por descubrimiento han sido tachadas de ser un tipo de «enfermedad infantil» del profesorado. Realmente, cuando se pregunta a los profesores en ejercicio o en formación qué se puede hacer para mejorar el aprendizaje en el aula, una de las primeras respuestas es que se debe aumentar la participación de los alumnos en actividades prácticas (Gil, 1994, Campanario, 1998a). Existe la creencia ingenua entre los profesores de ciencias de que la mera actividad práctica por sí misma puede conseguir efectos radicales en el aprendizaje de los alumnos.

A pesar de sus muchas limitaciones, el enfoque del aprendizaje por descubrimiento tiene algunos aspectos positivos aprovechables en la enseñanza de las ciencias experimentales. Por una parte, se insiste en el papel de 
los alumnos como responsables de su propio aprendizaje. Se presta, además, cierta atención a un aspecto del trabajo científico que a menudo había sido olvidado en la enseñanza tradicional de las ciencias: el aprender a descubrir. Este aspecto todavía constituye una de las más graves carencias de la formación en ciencias (Campanario, 1996; Lenox, 1985, Bavelas; 1987). Hay que tener en cuenta que no pocos descubrimientos científicos se deben a observaciones accidentales de fenómenos inesperados o a las consecuencias afortunadas de errores de procedimiento (Campanario, 1996; van Andel, 1994). Aprender a detectar anomalías debería ser, pues, uno de los objetivos educativos dignos de atención. Para que una observación pueda considerarse anómala es preciso conocer previamente qué cosa no resulta anómala; de ahí la relevancia de los conocimientos específicos. Numerosos ejemplos extraídos de la historia de la ciencia demuestran que ciertos hechos son considerados anómalos o dignos de ser explicados sólo en retrospectiva, es decir, cuando existe un marco conceptual en el que tales hechos desempeñan un determinado papel (Lightman y Gingerich, 1991). Como señala Polanyi, «ver un problema es una contribución significativa al conocimiento» (Birch, 1986, p. 79). No cabe duda de que el enseñar a los alumnos a observar con ojos críticos es quizás una de las aportaciones más dignas de consideración de una teoría del aprendizaje y la enseñanza que hoy día es casi unánimemente combatida por los especialistas en enseñanza de las ciencias, a veces, casi de oficio.

\section{LA ENSEÑANZA DE LAS CIENCIAS BASADA EN EL USO DE PROBLEMAS}

Desde puntos de vista pragmáticos se plantea basar, si no toda, la mayor parte de la enseñanza y el aprendizaje de las ciencias en el nivel universitario en la resolución de problemas por parte de los alumnos (Boud y Feletti, 1992). Como un ejemplo del vigor de esta línea de pensamiento pueden citarse los números especiales que las revistas Instructional Science y Journal of Education of the Gifted dedicaron en 1995 y 1997, respectivamente, a este tema. En esencia, la propuesta consiste en organizar unidades didácticas articuladas fundamentalmente como colecciones de problemas. El sistema no es tan simple como parece: los problemas han de ser seleccionados cuidadosamente y secuenciados de forma que se consiga el aprendizaje significativo (Lopes y Costa, 1996). La palabra problema debe ser entendida en un sentido amplio, ya que incluye, por ejemplo, pequeños experimentos, conjuntos de observaciones, tareas de clasificación, etc. Este enfoque deja entrever cierta influencia del método tradicional de aprendizaje del derecho en países anglosajones, basado en el análisis de casos concretos más que en el aprendizaje de códigos y sistemas completos y tiene como antecedente remoto las posturas filosóficas de John Dewey (Schmidt, 1995; Lopes y Costa, 1996). En el área de ciencias, el origen próximo de este enfoque se encuentra en la percepción de que gran parte de los conocimientos que tenían que aprender los estudiantes de medicina eran irrelevantes para su futuro desempeño profesional. La búsqueda de alternativas más prácticas desembocó en el aprendizaje a partir de problemas, un enfoque orientado típicamente a la enseñanza universitaria (Barrows y Tamblyn, 1980). La Facultad de Medicina de la Universidad McMaster en Ontario y el Worcester Polytechnical Institute fueron instituciones líderes en el uso de esta estrategia (Birch, 1986).

A pesar de que, como se ha indicado, este enfoque se plantea fundamentalmente para su aplicación en la enseñanza universitaria, es conveniente analizarlo con cierto detalle con el fin de aprovechar, en la medida de lo posible, las aportaciones que puedan ser de utilidad en la didáctica de las ciencias en los niveles de la enseñanza obligatoria. Según Schmidt, la propia dinámica interna de esta estrategia fomenta el aprendizaje autorregulado (Schmidt, 1995). Así, durante el análisis inicial del problema, el alumno debe crear un modelo mental relativo a la situación que se describe en el enunciado. Es muy posible que este primer modelo inicial sea incompleto y tenga lagunas importantes. Asimismo, descubrirá posibles alternativas y enfoques válidos que, en principio, pueden resultar apropiados para avanzar en la solución del problema o para explorar posibilidades. El que aprende debería entonces buscar y aprender contenidos relevantes. Cuando este enfoque se complementa con una organización cooperativa del trabajo en el aula, los problemas pueden hacerse más complejos y la búsqueda de información puede prolongarse durante más tiempo, a veces varios días o incluso una semana. Es evidente que en la formulación anterior gran parte de la responsabilidad del aprendizaje recae en el propio alumno.

Aunque aparentemente este enfoque puede tener ciertos rasgos superficiales comunes con el aprendizaje por descubrimiento, son más las diferencias que las semejanzas. Tal vez la diferencia más notable sea que con este método no se espera que el alumno descubra por sí mismo los conocimientos científicos. Más bien, la selección y sucesión de problemas le orienta para que apren$d a$, a partir de fuentes diversas, los contenidos que se estiman relevantes en una disciplina dada. El uso sistemático de los problemas estaría destinado a dar relevancia a tales contenidos, no a provocar su descubrimiento. Esta estrategia tiene también puntos en común con las concepciones constructivistas sobre el aprendizaje. Aunque los defensores del aprendizaje basado en problemas han argumentado que los puntos de vista recientes en psicología cognitiva son consistentes con el aprendizaje a partir de problemas, y existen justificaciones globales del modelo (Lopes y Costa, 1996), lo cierto es que la fundamentación epistemológica, filosófica y psicológica de esta orientación está menos elaborada que la de otros enfoques, como puede ser aprendizaje como investigación. Los defensores del aprendizaje a partir de problemas se basan, casi siempre, en evidencias relacionadas con el rendimiento académico y grado de motivación de los estudiantes.

Según Birch, el aprendizaje a partir de problemas es el mejor medio disponible para desarrollar las potencialidades generales de los alumnos (Birch, 1986). Este autor ha resumido las ventajas que se atribuyen al aprendizaje a partir de problemas. En primer lugar, el aprendizaje 
basado en problemas es más adecuado que los métodos tradicionales por transmisión para las necesidades de los alumnos, ya que entre las situaciones más frecuentes que se deben afrontar en las ciencias experimentales se encuentra la búsqueda de soluciones a situaciones problemáticas. Este aspecto es especialmente relevante en la enseñanza universitaria de cara a un futuro desempeño profesional. Dado que esta estrategia docente hace explícita la aplicación de los conocimientos teóricos a situaciones problemáticas, fomenta la percepción de la utilidad de los mismos, y contribuye, por tanto, a incrementar la motivación intrínseca. Dado que el alumno debe movilizar constantemente sus conocimientos y que existe una interrelación continua entre teoría y aplicación práctica, el aprendizaje basado en problemas puede conseguir una mejor integración de los conocimientos declarativos y procedimentales.

Como cualquier estrategia, el aprendizaje a partir de problemas presenta algunas limitaciones que es preciso tener en cuenta. Un posible inconveniente es que exige una mayor dedicación por parte del profesor. En efecto, la tarea del profesor no se reduce a seleccionar problemas que puedan ser más o menos compatibles con determinados contenidos teóricos. Por el contrario, la dirección en que se orienta el aprendizaje de los alumnos estaría determinada por la acertada selección de problemas y por la correcta secuenciación de los mismos. De la selección y secuenciación de los problemas depende además el interés que se logre despertar y el grado de coherencia interna que adquieren los contenidos que componen la asignatura. Se trata, además, de conseguir que el alumno convierta en suyos los problemas que elige el profesor como punto de partida del proceso de aprendizaje. Es evidente que esta estrategia exige prestar atención a los aspectos motivacionales y actitudinales de la enseñanza de las ciencias. El aprendizaje a partir de problemas requiere también mayor dedicación por parte del alumno y ello puede chocar con los hábitos pasivos de éstos, desarrollados tras años de inmersión en ambientes tradicionales.

Al igual que sucede con otras orientaciones educativas, el aprendizaje a partir de problemas es, más que una solución definitiva, una propuesta de trabajo y experimentación que merece sin duda un esfuerzo adicional de investigación. Entre los diversos aspectos del aprendizaje a partir de problemas que todavía no son bien comprendidos se pueden citar el papel que pueden desempeñar las nuevas tecnologías y la relación con factores personales propios de los alumnos, tales como la dependencia o independencia de campo (Birch, 1986). Por otra parte, las orientaciones sobre cómo desarrollar eficazmente este enfoque en la práctica son todavía objeto de vivo debate (Schmidt, 1995).

\section{EL CAMBIO CONCEPTUAL COMO PUNTO DE PARTIDA DE LAS IDEAS CONSTRUC- TIVISTAS}

Ante la evidente persistencia de las ideas previas de los alumnos y como una alternativa tanto a la enseñanza tradicional por transmisión como a la enseñanza por descubrimiento, diversos autores han planteado la búsqueda del cambio conceptual como punto de partida de las posiciones llamadas constructivistas (Driver, 1988; Nussbaum y Novick, 1982; Hewson y Hewson, 1984; Champagne, Klopfer y Gunstone, 1982). Desde estas posiciones se insiste en la necesidad de ofrecer oportunidades para que los alumnos expliciten sus ideas previas. En su ya clásico artículo, Posner, Strike, Hewson y Gertzog formulan su conocida concepción sobre el cambio conceptual y describen las condiciones necesarias para el mismo (Posner, Strike, Hewson y Gertzog, 1982):

a) Es preciso que exista insatisfacción con las concepciones existentes.

b) La nueva concepción debe ser inteligible, esto es, el alumno debe entender el modo en que la nueva concepción puede estructurar las experiencias anteriores.

c) La nueva concepción debe parecer inicialmente plausible. Esta condición es especialmente difícil de cumplir a veces, dado que algunas teorías científicas tienen aspectos que son contraintuitivos.

d) La nueva concepción debería ser útil, es decir, debería sugerir nuevas posibilidades de exploración y debería proporcionar nuevos puntos de vista al alumno. La nueva concepción debe resolver los problemas creados por su predecesora y explicar nuevos conocimientos y experiencias.

Esta visión del cambio conceptual en el aprendizaje de las ciencias se inspira en parte en las concepciones epistemológicas de Kuhn y Lakatos sobre el cambio conceptual en ciencia y en los puntos de vista de Toulmin sobre la evolución conceptual en ciencias en el marco de una ecología conceptual (Mellado y Carracedo, 1993). La propuesta inicial del cambio conceptual sería un modelo de las condiciones necesarias para un tipo de aprendizaje (Hewson y Beeth, 1995). Al ser una teoría descriptiva, no prescribe un modelo docente determinado. Sin embargo, a la vista de los numerosos intentos de llevar a la práctica esta estrategia, puede considerarse como una propuesta acerca de cómo debe orientarse la enseñanza.

En 1992, diez años después de su formulación inicial, Hewson señalaba el gran número de artículos publicados en revistas internacionales y de sesiones en congresos dedicados a la investigación sobre el cambio conceptual como un indicador del grado desarrollo que ha alcanzado este frente de investigación y práctica educativa en el área de enseñanza de las ciencias (Hewson, 1992). Además, como un indicador adicional de la robustez de este punto de vista, Hewson constataba el hecho conocido de que el cambio conceptual como paradigma se ha extendido a otras áreas e incluso hay documentos de la reforma educativa en España que se refieren al aprendizaje como un cambio conceptual en la estructura cognitiva del alumno y proponen como uno de los objetivos de la enseñanza de las ciencias el propiciar cambios en las ideas previas de los alumnos. La posiciones que abogan 
por el cambio conceptual conciben el currículo como un conjunto de experiencias mediante las cuales el alumno construye una concepción del mundo más cercana a la concepción de los científicos (Driver, 1988). En general, las estrategias que promueven el cambio conceptual reflejan un estilo de enseñanza en el cual tanto alumnos como profesores están implicados activamente y en el que los profesores «animan a los alumnos a expresar sus ideas, a pensar rigurosamente $\mathrm{y}$, a su vez, modifican sus explicaciones dependiendo de los puntos de vista que consiguen elicitar en sus alumnos» (Smith, Blakeslee y Anderson, 1993, p. 114). Las pautas generales que deberían seguirse en cualquier programa de enseñanza para el cambio conceptual han sido revisadas recientemente por Hewson y Beeth, quienes ofrecen una serie de recomendaciones que se resumen a continuación (Hewson y Beeth, 1995):

a) Las ideas de los alumnos deberían ser una parte explícita del debate en el aula. Se trata de que los alumnos sean conscientes de sus propias ideas y de las ideas de los demás. Además, a diferencia de los enfoques tradicionales, las opiniones de los alumnos deberían considerarse al mismo nivel que las del profesor. Los alumnos han de darse cuenta de que las ideas tienen autoridad por su poder explicativo, no por la fuente de donde proceden.

b) El estatus de las ideas tiene que ser discutido y negociado. Como una consecuencia de la primera condición, una vez que todas las ideas han sido elicitadas, los alumnos deben decidir acerca del estatus de sus propias opiniones y de las opiniones de los demás. En esta elección intervienen, además de la propia ecología conceptual, sus criterios epistemológicos acerca del conocimiento científico y acerca de qué constituye una explicación aceptable.

c) La justificación de las ideas debe ser un componente explícito del plan de estudios. Que los alumnos consideren que las nuevas concepciones son plausibles y útiles puede depender de varios factores: que las nuevas concepciones parezcan verdaderas y compatibles con otras concepciones previas o aprendidas, que las concepciones no contradigan las ideas metafísicas de los alumnos, que la idea aparezca como general o como consistente y que ello coincida con los compromisos epistemológicos de los alumnos, etc.

d) El debate en el aula debe tener en cuenta la metacognición que, según Gunstone y Northfield, desempeña un papel central en el cambio conceptual (Gunstone y Northfield, 1994). Cuando los alumnos comentan, comparan y deciden sobre la utilidad, la plausibilidad y la consistencia de las concepciones que se presentan, están explicitando sus propios criterios de comprensión. La aceptación o no de las nuevas ideas y el rechazo de las ideas previas depende en gran medida de los patrones metacognitivos de los alumnos: ¿satisface una nueva concepción las lagunas que plantea la anterior?, ¿es capaz el alumno de detectar fallos en la capacidad explicativa de sus propias ideas?, ¿cómo comparar el poder explicativo, sin duda elevado, de las concepciones previas con el de las nuevas concepciones, etc.? Los interrogantes anteriores responden a la necesidad de tener en cuenta problemas como las dificultades de los alumnos para detectar discrepancias o inconsistencias en un razonamiento científico (Otero y Campanario, 1990).

De la descripción anterior se desprende la necesidad de disponer de un repertorio de técnicas y recursos acordes con las condiciones que se han explicado. Las ideas previas pueden ponerse de manifiesto utilizando ejemplos adecuados, cuestionarios, demostraciones, técnicas de discusión en grupo, etc. Una vez que se ha conseguido lo anterior, las estrategias para disminuir el estatus de las ideas erróneas de los alumnos y para justificar las nuevas ideas deben hacer hincapié en los principios científicos de buscar la máxima simplicidad o la máxima consistencia; se basan en el empleo, entre otros recursos, de analogías (Brown, 1994), discusiones guiadas, modelizaciones (Raghavan y Glaser, 1995), comparaciones, etc. El uso de estas actividades incide, además, sobre las concepciones epistemológicas de los alumnos. Por último, es necesario enseñar a los alumnos a detectar inconsistencias entre diversos puntos de vista (algo que se da por supuesto, aunque no siempre esté garantizado) y a que aprendan a aplicar criterios de comprensión adecuados en tales situaciones.

En la literatura existen numerosos ejemplos de aplicación de los principios generales del cambio conceptual a áreas diversas y a temas y contenidos concretos. Osborne y Freyberg han descrito varios modelos consecuentes con esta orientación (Osborne y Freyberg, 1985). La revista española Enseñanza de las Ciencias es una fuente notable de este tipo de propuestas. Aunque no parecen existir revisiones sistemáticas o metaanálisis comprensivos de los resultados, los trabajos publicados demuestran cierto grado de efectividad, sin que los resultados sean espectaculares (Carretero y Limón, 1995; Linder, 1993). Parece ser que, como reconocen Strike y Posner (1990), las ideas previas pueden resistir incluso a la enseñanza que se propone explícitamente erradicarlas.

Las causas de que el éxito no siempre acompañe a los intentos de conseguir el cambio conceptual son variadas. Algunos críticos señalan que el fundamento epistemológico basado en las ideas de Toulmin y Kuhn puede ser útil para entender los procesos de cambio conceptual en poblaciones de científicos. Sin embargo, la aplicación casi directa de tales ideas para entender los cambios individuales en la mente de los alumnos es más que discutible (Pintó, Aliberas y Gómez, 1996; Osborne, 1996). Además, el efecto de las evidencias contrarias a las ideas previas de los alumnos de cara a lograr el cambio conceptual parece ser menor del que se pensaba en un principio, de manera que los contraejemplos o los conflictos cognitivos por sí mismos no siempre son útiles para provocar el cambio conceptual (Carretero y Baillo, 1995; Clement, Brown y Zietsman, 1989; Hewson y Thorley, 1989). Este aspecto es relevante para el profesor de ciencias porque algunos sesgos en el funcionamiento cognitivo, comunes a casi todos adultos, hacen que los alumnos sean selectivos cuando interpretan las 
observaciones experimentales, lo que podría explicar la relativa falta de eficacia de los contraejemplos como único recurso para cuestionar sus ideas previas. Además, parece claro que ni siquiera los propios científicos utilizan la falsación simple para rechazar un punto de vista (Chalmers, 1982). De hecho, los científicos hacen gala a veces de una resistencia notable a las nuevas ideas en ciencia (Campanario, 1993, 1997).

Entre los investigadores en enseñanza de las ciencias existe la percepción de que para que las estrategias de cambio conceptual tengan algún efecto importante es preciso que no se apliquen como un conjunto de estrategias aisladas, sino como un enfoque de enseñanza coherente. Para ello sería necesaria, en primer lugar, una orientación común en varias asignaturas de ciencias y una cierta persistencia temporal en cada una de ellas (Smith, Blakeslee y Anderson, 1993). Además, mientras los profesores tienen una perspectiva a largo plazo de las actividades de enseñanza, los alumnos no tienen, en general, esta perspectiva (Duit, 1991). Es aconsejable, pues, que el profesor explicite los objetivos de las actividades de enseñanza-aprendizaje. Por otra parte, aunque las estrategias de cambio conceptual den resultado en casos concretos, los profesores reconocen que no pueden aplicar este enfoque a gran escala sin el apoyo de materiales curriculares adecuados (Smith, Blakeslee y Anderson; 1993). Los libros de texto son el material curricular más utilizado en la enseñanza a todos los niveles y sólo en raras ocasiones incorporan esta orientación didáctica (Apple, 1984).

Las formulaciones iniciales del cambio conceptual se centran casi exclusivamente en los conocimientos. Ciertamente, este enfoque parece destinado a sustituir las ideas previas de los alumnos por otras concepciones acordes con las comúnmente aceptadas por los científicos como un fin en sí mismo. Aunque en formulaciones posteriores del cambio conceptual se destaca la importancia de otros factores, como los compromisos epistemológicos (Hewson, 1985) y los factores afectivos y estéticos (West y Pines, 1983; Hewson y Thorley, 1989), y metacognitivos (Hewson, 1992), el marco anterior no presta, en principio, excesiva atención a otras variables relevantes y aún considera que los nuevos elementos añadidos deben servir casi exclusivamente como ayuda para el cambio conceptual. Sin embargo, los factores afectivos son importantes e incluso decisivos. Por ejemplo, Dreyfus, Jungwirth y Eliovitch han comprobado que las condiciones de conflicto cognitivo son bien recibidas por los alumnos más brillantes, mientras los alumnos con dificultades de aprendizaje pueden llegar a desarrollar actitudes negativas y a dar muestras de ansiedad ante tales situaciones (Dreyfus, Jungwirth y Eliovitch, 1990). Si no se tienen en cuenta estos factores, es posible que las estrategias de cambio conceptual no sean efectivas.

El papel de la metacognición en el cambio conceptual es especialmente relevante desde otra perspectiva. Por una parte es un medio para que tenga lugar el cambio conceptual, pero también puede considerarse un resultado deseable del cambio conceptual. La insatisfacción del alumno con sus propias concepciones implica el recono- cimiento de dificultades (Gunstone y Northfield, 1994), mientras que la evaluación de las nuevas concepciones para decidir sobre la plausibilidad de las mismas y su utilidad implica comparación entre dos estados de comprensión. En esta comparación juegan un papel determinante los compromisos epistemológicos que tenga el alumno. Possner, Strike, Hewson y Gertzog llaman compromisos epistemológicos a los criterios mediante los que una persona utiliza y juzga el conocimiento (Posner, Strike, Hewson y Gertzog, 1982). Un alumno puede tener compromisos epistemológicos que enfaticen la coherencia interna o la generalidad del conocimiento. Enfrentado a la tarea de reconocer el conflicto entre dos concepciones inconsistentes, un alumno puede elegir entre admitir las dos versiones como correctas cada una dentro de su propio dominio o rechazar una de las dos concepciones. Es evidente que en todo el proceso anterior el alumno necesita controlar constantemente el estado actual de la propia comprensión. Si se consigue que los alumnos sean conscientes del carácter constructivo del aprendizaje, el cambio conceptual puede ser un medio para fomentar la metacognición.

Movidos, en parte, por las limitaciones anteriores, algunos autores hablan del cambio conceptual y metodológico (Carrascosa y Gil, 1985; Gil, 1994; Segura, 1991). Ciertamente, la inclusión de este último término es algo más que un mero complemento: se argumenta que sin un cambio metodológico no puede producirse un cambio conceptual. De ahí que, según estos puntos de vista, los enfoques que sólo tienen en cuenta el cambio conceptual resulten, por fuerza, limitados.

\section{EL APRENDIZAJE DE LAS CIENCIAS PUEDE SER UN PROCESO DE INVES- TIGACIÓN DIRIGIDA}

Aunque la concepción del aprendizaje como un proceso de investigación no es nueva, en los últimos años las propuestas coherentes con esta idea han adquirido un desarrollo notable, especialmente desde posiciones llamadas constructivistas. García y Cañal han revisado las propuestas que defienden el aprendizaje como investigación más difundidas en nuestro país y han encontrado una serie de componentes comunes en todas ellas y algunas diferencias de matiz (García y Cañal, 1995). El lector interesado en un análisis comparativo de las diferentes propuestas puede consultar la obra citada. A continuación se analiza brevemente una de las formulaciones más difundidas en España y de la cual el profesor Daniel Gil es quizá el más conocido defensor.

Según Gil, uno de los mayores problemas de la enseñan$\mathrm{za}$ de las ciencias es el abismo que existe entre las situaciones de enseñanza-aprendizaje y el modo en que se construye el conocimiento científico (Gil, 1994). En consecuencia, es útil partir de la metáfora del científico novel que, como es sabido, puede alcanzar en un tiempo más o menos corto un grado de competencia relativamente elevado en un dominio concreto. Ello es posible porque cuando un científico novel se integra en un grupo 
de investigación empieza a desarrollar pequeñas investigaciones en las que replica los trabajos previos en un área determinada y aborda problemas en los que sus supervisores son expertos. De este planteamiento se desprende la conveniencia y aun la necesidad de plantear el aprendizaje de las ciencias como una investigación dirigida de situaciones problemáticas de interés (Gil, 1993). Esta propuesta se orienta, fundamentalmente, a la enseñanza de la ciencia en el nivel de enseñanza secundaria, si bien en la literatura didáctica existen ejemplos de aplicación orientados a la enseñanza universitaria (Meneses, 1992).

Gil y sus colaboradores proponen una serie de estrategias que se detallan a continuación sin que ello implique la necesidad de seguir forzosamente una secuencia predeterminada (Gil, 1993; Gil, 1994, Gil, Carrascosa, Furió y Martínez-Torregrosa; 1991):

a) Se plantean situaciones problemáticas que generen interés en los alumnos y proporcionen una concepción preliminar de la tarea.

b) Los alumnos, trabajando en grupo, estudian cualitativamente las situaciones problemáticas planteadas $\mathrm{y}$, con las ayudas bibliográficas apropiadas, empiezan a delimitar el problema y a explicitar ideas.

c) Los problemas se tratan siguiendo una orientación científica, con emisión de hipótesis (y explicitación de las ideas previas), elaboración de estrategias posibles de resolución y análisis y comparación con los resultados obtenidos por otros grupos de alumnos. Es ésta una ocasión para el conflicto cognitivo entre concepciones diferentes, lo cual lleva a replantear el problema y a emitir nuevas hipótesis.

d) Los nuevos conocimientos se manejan y aplican a nuevas situaciones para profundizar en los mismos y afianzarlos. Éste es el momento más indicado para hacer explícitas las relaciones entre ciencia, tecnología y sociedad.

Como señala Gil, el cambio conceptual adquiere ahora un carácter instrumental y deja de ser un objetivo en sí mismo: «la investigación no se plantea para conseguir el cambio conceptual, sino para resolver un problema de interés» (Gil, 1994, p. 27), el cambio conceptual «se produce a lo largo de todo el proceso como un resultado más» (Gil, 1993, p. 203), de ahí el énfasis en el necesario cambio metodológico que debe acompañar todo el proceso. Sin embargo, se rechaza la idea de reducir todo al aprendizaje de un método científico «como conjunto de reglas perfectamente definidas que se aplican mecánicamente» (Gil, 1983, p. 26). Ésta y otras formulaciones insisten también en el cambio actitudinal (Mellado y Carracedo, 1993, p. 335).

El modelo que emerge del aprendizaje de las ciencias aparece así contrapuesto tanto a la mera recepción de conocimientos como al descubrimiento de los mismos por los alumnos (Gil, 1993). Por otra parte, según los defensores de este enfoque, tanto los diseñadores del currículo como los profesores deben cuestionar la ciencia que se debe (y que es posible) enseñar. Concretamente, es preciso descargar los programas de ciencias de contenidos puramente conceptuales y prestar más atención a los aspectos metodológicos, al estudio de la naturaleza del conocimiento científico, a los procesos de construcción del mismo y a la relación ciencia-tecnología-sociedad (Gil, 1994).

Las estrategias propias del aprendizaje como investigación deben ir acompañadas por actividades de síntesis que den lugar a la elaboración de productos como esquemas, memorias, mapas conceptuales, etc., y que permitan concebir nuevos problemas. Coherente con este enfoque, la resolución de problemas como investigación se propone como alternativa a los problemas y ejercicios tradicionales (Gil, Martínez-Torregrosa y Senent, 1988). En formulaciones recientes del modelo, se insiste en cuestionar la separación tradicional entre prácticas, resolución de problemas y teoría y se ofrecen alternativas de integración concretas (Gil y Valdés, 1995).

Como se ha explicado más arriba, los puntos de vista que proponen la investigación como medio de enseñanza y aprendizaje no son nuevos, y autores clásicos como Locke, Rousseau, Ferrer i Guardia y Dewey ya formularon propuestas en tal sentido (García y Cañal, 1995). Sin embargo, tal vez los rasgos más distintivos de los enfoques actuales del aprendizaje como investigación sean su afán integrador de los diversos aprendizajes (Gil, 1993) y su orientación «radicalmente constructivista» (Gil, 1994, p. 29) fundamentada en las teorías y puntos de vista actuales en filosofía, historia y epistemología de la ciencia. Los autores que trabajan desde estas perspectivas constructivistas han consolidado una línea de investigación y práctica escolar sólida y fructífera. Si nos ceñimos a nuestro país, basta un análisis somero de revistas como Enseñanza de las Ciencias o Investigación en la Escuela para constatar el número elevado de trabajos que se publican siguiendo esta orientación teórica. En una interesante revisión sobre las tendencias actuales en la formación del profesorado de ciencias, Furió abogaba por la integración de las nuevas exigencias prácticas de una enseñanza constructivista (Furió, 1994). Sin embargo, como señalan Mellado y Carracedo, «el constructivismo se ha mostrado como un paradigma coherente y fundamentado para el aprendizaje de las ciencias, pero no puede considerarse como un paradigma dominante único, al estilo kuhniano, que excluya absolutamente a los demás» (Mellado y Carracedo, 1993, p. 336).

Al igual que sucede con otros enfoques, el aprendizaje como investigación no está exento de problemas. En su aplicación práctica existen algunas dificultades que es preciso tener en cuenta. Una de estas dificultades tiene que ver con la capacidad investigadora de los alumnos. La metáfora del alumno como científico ha sido cuestionada por autores que llaman la atención sobre las pautas sesgadas de razonamiento que utilizan con frecuencia los alumnos (Thiberghien, Psillos, Koumaras, 1995). Ello obliga casi siempre a plantear situaciones muy simplificadas y a que el profesor deba anticipar muchas 
de las dificultades conceptuales y de procedimiento que, sin duda, surgirán durante el desarrollo de las clases. De ahí el marcado carácter de investigación dirigida que presenta este enfoque. De hecho, como reconoce Gil, no resultará extraño que el profesor deba reforzar, matizar o poner en cuestión los resultados obtenidos por los alumnos mediante los resultados «correctos» obtenidos por los científicos (Gil, 1994, p. 29). Por otra parte, el desarrollo de las actividades de investigación dirigida exige bastante tiempo y obliga, en cierta medida, a un delicado equilibrio entre las necesidades contrapuestas de profundización y visión coherente y ello exige con frecuencia el sacrificio de parte de los contenidos (Gil, 1987). Otro riesgo no desdeñable tiene que ver con la actitud de los alumnos. Al igual que sucede con otros enfoques innovadores, es posible que los alumnos no estén dispuestos a realizar la inversión de esfuerzo que conlleva un modo de aprender distinto al que generalmente están acostumbrados. Muchas veces es más cómodo para los alumnos recibir explicaciones o puede que no encuentren interesantes las situaciones que se abordan en el trabajo de investigación. Como señala Gil, los inconvenientes anteriores no son desdeñables e inciden negativamente en el desarrollo de las actividades de clase, aunque ello tiene una contraparte positiva y es que, en cierta medida, «se evitan las expectativas simplistas en soluciones-milagro»(Gil, 1987, p. 12).

\section{LA ENSEÑANZA DE LAS CIENCIAS Y EL DESARROLLO DE LAS CAPACIDADES METACOGNITIVAS}

Como se ha indicado más arriba, las destrezas metacognitivas son una de las componentes del aprendizaje a las que se ha empezado a prestar atención en los últimos años. La metacognición puede concebirse como una ayuda al aprendizaje, pero también puede y debe constituir un objetivo legítimo de la enseñanza (Novak y Gowin, 1988). Se ha argumentado incluso que la enseñanza de las ciencias puede resultar especialmente adecuada para este propósito (Baker, 1991). En la literatura educativa se citan diversas propuestas en este sentido, algunas de las cuales se revisan a continuación.

Linda Baker y otros autores han llamado la atención sobre la relación que existe entre una de las componentes de la metacognición, el uso de estrategias metacognitivas, y otros aspectos relacionados con el aprendizaje de las ciencias (Baker, 1991; Carin y Sand, 1985; Carter y Simpson, 1978; Esler y Esler, 1985; Resnick, 1983). Entre las destrezas básicas que se espera que desarrollen los alumnos de ciencias destacan las capacidades de observación, clasificación, comparación, medición, descripción, organización coherente de la información, predicción, formulación de inferencias e hipótesis, interpretación de datos, elaboración de modelos, y obtención de conclusiones (Esler y Esler, 1985; Carter y Simpson, 1978). Según Baker, existe un paralelismo notable entre algunas de las destrezas básicas anteriores y ciertas estrategias cognitivas y metacognitivas que se necesitan y aplican en el procesamiento de información. Cuando el procesamiento de la información se lleva a cabo a partir de textos, el paralelismo es aún más claro. Así, la formulación de inferencias en el laboratorio de ciencias requiere, en primer lugar, que el alumno tenga en cuenta toda la información disponible en cada momento. Esta información puede ser fragmentaria, contradictoria o incompleta, lo cual muchas veces se debe a limitaciones experimentales. El alumno debe formular determinadas predicciones que le ayuden a completar la información de que dispone y, como paso final, debe comprobar que las inferencias eran apropiadas. En todos los pasos anteriores existe un paralelismo con los procesos de formulación y comprobación de inferencias durante las tareas de lectura de textos. El aprendizaje a partir de textos se postula, pues, como uno de los medios más eficaces de fomentar la metacognición, especialmente en el aprendizaje de las ciencias (Baker, 1991).

Se han desarrollado algunos programas de enseñanza explícita de la metacognición como un contenido educativo más. La idea que subyace es que los alumnos poseen las capacidades necesarias para aplicar destrezas metacognitivas, pero con frecuencia no son capaces de hacerlo de manera espontánea. La mayor parte de los programas de instrucción directa en capacidades metacognitivas que se han publicado se destinan a mejorar el aprendizaje a partir de textos (Campanario, 1995) y no están orientados explícitamente al aprendizaje de las ciencias (Campanario, en revisión).

Precisamente, la escasez de propuestas para desarrollar las capacidades metacognitivas en el marco general del aprendizaje de las ciencias es un rasgo común a los enfoques orientados al cambio conceptual o al aprendizaje como investigación, a pesar de la importancia que se concede actualmente a la metacognición en dichas propuestas. Una forma posible de desarrollar la metacognición en el marco del cambio conceptual consiste en el empleo de actividades que siguen el esquema predecirobservar - explicar (Gunstone y Northfield, 1994). En estas actividades se hace que los alumnos formulen, en primer lugar, predicciones acerca de determinadas experiencias o demostraciones de cátedra. Se pone especial atención en que los alumnos expliciten las razones en que se basan para sus predicciones. El objetivo es que los alumnos sean conscientes del papel de los conocimientos previos en la interpretación de los fenómenos. A continuación se desarrolla la experiencia para que los alumnos contrasten el desarrollo y los resultados de la misma con sus predicciones. Por último, los alumnos deben intentar explicar las observaciones realizadas, que muchas veces serán distintas a sus predicciones. A lo largo de este proceso, el profesor debe hacer explícitas las relaciones entre las ideas previas de los alumnos y las teorías que permiten explicar adecuadamente las observaciones realizadas durante las experiencias. Como señalan Gunstone y Northfield, este tipo de actividades tiene un marcado carácter metacognitivo en la medida en que, si se desarrollan adecuadamente, ayudan a los alumnos a ser conscientes de sus propios procesos cognitivos (Gunstone y Northfield, 1994). Que los alumnos comprendan que los conocimientos previos guían la observación ya es un objetivo valioso en sí mismo y lo es 
más si contribuye a que sean conscientes de que sus concepciones sobre el conocimiento científico suelen ser inadecuadas. Este tipo de actividades ha sido utilizado con éxito con profesores en formación y con profesores en servicio (Gunstone y Northfield, 1994). Otra ventaja de este tipo de tareas es que con ellas se llama la atención sobre el papel de la observación en ciencia: no basta con dar por supuestos los resultados, es preciso contrastarlos.

Otra estrategia, si bien a largo plazo, consiste en hacer que los alumnos lleven un diario de campo en el que registren las experiencias realizadas en clase, sus concepciones iniciales y los procesos de cambio conceptual. De esta manera, a medida que se avanza en el desarrollo de las asignaturas, existe una base documental a la que se puede recurrir para fomentar la autoevaluación por parte de los alumnos del cambio en sus concepciones sobre el aprendizaje (Fulwiler, 1987).

Aunque no están únicamente orientados al desarrollo de la metacognición, los mapas conceptuales y los diagramas UVE pueden servir para este objetivo y a menudo se presentan como dos recursos realmente útiles tanto para el aprendizaje de los contenidos como para el desarrollo de las capacidades metacognitivas (Novak y Gowin, 1988).

\section{EL DISEÑO DE UNIDADES DIDÁCTICAS PARA LA ENSEÑANZA DE LAS CIENCIAS}

La preparación de las clases constituye una tarea que ha de acometer diariamente el profesor. La preparación de una clase conlleva la elección de los contenidos, la organización y secuenciación de los mismos, el diseño de actividades de clase y de posibles tareas extraescolares, la anticipación de las dificultades que pueden encontrar los alumnos, etc. Todos estos componentes se traducen, en definitiva, en una secuencia determinada de acciones. Como señalan García y Cañal, es indispensable complementar los enfoques «macro» (de orientación teórica y que proporcionan modelos de enseñanza generales) con otros de tipo «micro» (más orientados a la acción) que, en definitiva, implementen los modelos generales de enseñanza en la dinámica del aula y en las actividades de enseñanza (García y Cañal, 1995). De ahí que las diversas concepciones sobre la enseñanza y aprendizaje ofrezcan recomendaciones concretas para secuenciar las actividades de enseñanza de acuerdo con sus postulados (Lledó y Cañal, 1993).

Sánchez y Valcárcel han presentado una serie detallada de recomendaciones para el diseño de unidades didácticas en el área de ciencias experimentales (Sánchez y Valcárcel, 1993). El modelo de estos autores incluye cinco componentes: análisis científico, análisis didáctico, selección de objetivos, selección de estrategias didácticas y selección de estrategias de evaluación. En el trabajo citado, los autores detallan los objetivos y proponen procedimientos para cada una de las componentes anteriores. Así, por ejemplo, para el análisis científico se requiere un proceso de selección de contenidos y de delimitación de los esquemas conceptuales, de los procedimientos científicos y de las actitudes. En el análisis didáctico hay que averiguar las ideas previas de los alumnos, analizar las exigencias cognitivas de los contenidos y delimitar las implicaciones para la enseñanza. Para la selección de estrategias didácticas, otro de los componentes del modelo, los autores sugieren el diseño de una secuencia global de enseñanza, la selección de actividades de enseñanza y la elaboración de materiales de aprendizaje.

Los programas-guía de actividades representan otra aplicación del modelo constructivista de aprendizaje de las ciencias (Gil, 1987). Las ideas básicas que subyacen en la elaboración de estos programas-guía son favorecer la construcción de los conocimientos por parte de los alumnos y lograr que se familiaricen con algunas características del trabajo científico. Los programas-guías son propuestas de desarrollo de unidades didácticas y, aunque deben ser cuidadosamente preparados, han de estar abiertos a posibles modificaciones a la vista de los resultados que se obtengan durante su aplicación. Sería contrario a la orientación constructivista utilizar los programas-guía como una receta inflexible de la que no se puede salir. Los programas-guía describen una secuencia de enseñanza en términos genéricos, relacionando el conjunto de actividades que se incluyen en ella y posibles alternativas de trabajo adicionales. Las actividades que conforman los programas-guía pueden ser muy variadas, pero se pueden clasificar en tres categorías fundamentales (Gil, 1987): actividades de iniciación (sensibilización del tema, explicitación de las ideas que posean los alumnos, etc.), actividades de desarrollo (introducción de conceptos científicos, manejo reiterado de dichos conceptos, detección de errores, emisión y fundamentación de hipótesis, conexión entre partes distintas de la asignatura, elaboración de diseños experimentales, etc.) y actividades de acabado (elaboración de síntesis, esquemas, mapas conceptuales, evaluación del aprendizaje, etc.). Como se puede apreciar, algunas de las actividades tienen una marcada orientación metacognitiva.

La forma en que se utiliza el programa-guía consiste en la realización ordenada por los alumnos de las actividades propuestas. Los alumnos abordan las actividades que se plantean en el programa-guía trabajando en grupos pequeños. De esta manera se incrementa el nivel de participación y la motivación de los alumnos. El profesor debe supervisar el trabajo de los grupos, ofrecer ayudas puntuales cuando sea necesario, estar atento al desarrollo de las tareas y, tras la realización de cada actividad, coordinar la puesta en común y reformular los resultados, a la vez que clarifica y complementa el trabajo de los grupos. Los programas-guía se orientan fundamentalmente a la enseñanza secundaria.

En una línea diferente, pero complementaria, Villani y Orquiza abogan por el uso de experimentos cualitativos en las unidades didácticas como medio de crear conflictos cognitivos en los alumnos (Villani y Orquiza, 1995). Estos autores clasifican los conflictos en externos e 
internos. Los conflictos externos tienen su origen en una divergencia entre los modos de ver de los alumnos y elementos externos a él, como son el resultado de un experimento, los contenidos de un libro de texto o la explicación de un profesor, mientras que los conflictos internos tienen su origen en una divergencia entre elementos cognitivos de los alumnos, como sus ideas, sus exigencias epistemológicas o cognitivas o su ecología conceptual. Villani y Orquiza insisten en que los alumnos no siempre son conscientes de que existe un conflicto externo o interno, algo que hemos podido comprobar también en nuestras propias investigaciones (Otero y Campanario, 1990; Otero, Campanario y Hopkins, 1992; García-Arista, Campanario y Otero, 1996; Campanario, 1995). Otras veces los alumnos niegan, deforman o minimizan los elementos divergentes. Los autores aconsejan estrategias tales como evitar el exceso de información, estimular las preguntas de los alumnos, soslayar los problemas poco maduros, distinguir las situaciones problemáticas, insistir en las condiciones de validez de las afirmaciones de los alumnos, etc.

\section{CONCLUSIONES}

La revisión anterior ofrece un abanico de posibilidades para el profesor de ciencias que no excluye otras propuestas alternativas (por ejemplo, el análisis y aplicación de las estrategias de enseñanza que utilizan los profesores modelo (Tobin y Fraser, 1990) o la enseñanza de las ciencias basada en una orientación histórica (Lombardi, 1997; Campanario, 1998b)). Los distintos enfoques presentan diferencias evidentes entre sí, y quizá sea la fundamentación psicológica, filosófica o epistemológica uno de los aspectos que diferencia las propuestas. Sin embargo, las orientaciones revisadas también tienen aspectos comunes o similares que vale la pena resaltar.

En primer lugar, los enfoque alternativos a la enseñanza tradicional insisten en la necesidad de que los alumnos desempeñen un papel más activo en clase. Esta actividad puede consistir en tareas diversas, desde realizar experiencias hasta resolver problemas, y se concibe como una elaboración o aplicación de los conocimientos que constituya una alternativa a la memorización simple de los mismos.

Las propuestas que se revisan en este trabajo son consistentes, a veces de forma implícita, con algunas de las recomendaciones basadas en las teorías cognitivas sobre la motivación intrínseca de los sujetos. En efecto, uno de los rasgos que definen el interés intrínseco por una tarea o un contenido es la aplicabilidad percibida del mismo y su utilidad para resolver o entender problemas o situaciones de interés (Alonso Tapia, 1991), un rasgo común a los enfoques anteriores.
Por otra parte, parece existir un cierto consenso en que estas propuestas requieren, en general, más tiempo para desarrollar los contenidos que el que se requiere en la enseñanza tradicional. La consecuencia inmediata de esta percepción es una recomendación para reducir los programas de las asignaturas.

La metacognición comienza a ser tenida en cuenta explícitamente en algunos de los enfoques, que hacen de este factor un elemento importante de su formulación. Desgraciadamente, no parece que todavía existan muchas propuestas para incidir sobre este factor orientadas a la enseñanza de las ciencias y que estén debidamente contrastadas (Campanario, en revisión).

Los defensores de algunas de las alternativas revisadas coinciden en señalar que una parte de las dificultades derivadas de la implementación de las mismas tienen su origen en las resistencias previsibles por parte de los alumnos o de los profesores. Los profesores y las autoridades educativas tienden a ser conservadores a la hora de aceptar e implementar las nuevas propuestas. Así, por ejemplo, en otros países, el mayor impedimento al progreso del aprendizaje basado en problemas «ha sido el conservadurismo y la resistencia a la innovación» (Birch, 1986, p. 74). No cabe duda de que un profesor decidido a aplicar alguna de las estrategias de enseñanza alternativas que se proponen en este artículo debe reconsiderar cuál es su papel en el aula, lo que se traducirá, sin duda, en un menor protagonismo y ello puede interpretarse, erróneamente, como una cierta merma en su autoridad.

Las consideraciones anteriores nos llevan al arduo problema de la formación de los profesores de ciencias. La formación psicopedagógica inicial de los profesores de enseñanza secundaria en España es deficiente en muchos aspectos. Además de una reforma en profundidad de esta formación inicial que contribuya a eliminar las ideas «de sentido común» sobre la enseñanza, sería necesario inculcar en los futuros profesores la necesidad de una formación continuada. Esta formación continua implica una transformación continua. Creemos que un buen profesor debe conocer, además de su disciplina, los puntos de vista vigentes sobre la enseñanza de las ciencias para, tras un análisis crítico, adaptar aquello que encuentre valioso, corregir lo que sea deficitario y aportar, en un proceso de experimentación continuado, nuevas ideas y puntos de vista.

\section{AGRADECIMIENTOS}

Este trabajo fue realizado en el marco del proyecto de investigación PB-93-0478 financiado por la DGICYT. Expresamos nuestro agradecimiento a tres asesores anónimos de la revista Enseñanza de las Ciencias por sus sugerencias y comentarios. 


\section{REFERENCIAS BIBLIOGRÁFICAS}

ALONSO TAPIA, J. (1991). Motivación y aprendizaje en el aula. Madrid: Santillana, Aula XXI.

APPLE, M. (1984). Economía de la publicación de libros de texto. Revista de Educación, 275, 43-62.

AUSUBEL, D.P., NOVAK, J.D. y HANESIAN, H. (1983). Psicología educativa: Un punto de vista cognitivo. México: Editorial Trillas.

BAKER, L. (1991). Metacognition, reading and science education, en Santa, C.M. y Alvermann, D. (eds.), Science learning: Processes and applications. Newsdale, Delaware: International Reading Association.

BARROWS, H.S. y TAMBLYN, R.M. (1980). Problem-based learning. Nueva York: Springer Verlag.

BAVELAS, J.B. (1987). Permitting Creativity in Science, en Jackson, D.N. y Rushton, J.P. (eds.). Scientific Excellence. Newbury Park: Sage.

BIRCH, W. (1986). Towards a model for problem-based learning. Studies in Higher Education, 11, pp. 73-82.

BOUD, D. y FELETTI, G. (1992) The challenge of problembased learning. Londres: KoganPage.

BROWN, D.E. (1994). Facilitating conceptual change using analogies and explanatory models. International Journal of Science Education, 16, pp. 201-214.

CALATAYUD, M.L., GIL, D. y GIMENO, J.V. (1992). Cuestionando el pensamiento docente espontáneo del profesorado universitario: ¿Las deficiencias en la enseñanza como origen de las dificultades de los estudiantes? Revista Interuniversitaria de Formación del Profesorado, 14, pp. 71-81.

CAMPANARIO, J.M. (en revisión). Metacognición y aprendizaje de las ciencias: ¿cómo fomentar el uso de estrategias metacognitivas?

CAMPANARIO, J.M. (1993). Consolation for the scientist: Sometimes it is hard to publish papers that are later highlycited. Social Studies of Science, 23, pp. 342-362.

CAMPANARIO, J.M. (1995). Los problemas crecen: a veces los alumnos no se enteran de que no se enteran. Aspectos didácticos de Física y Química (Física), 6, pp. 87-126. Zaragoza: ICE. Universidad de Zaragoza.

CAMPANARIO, J.M. (1996). Using Citation Classics to study the incidence of serendipity in scientific discovery. Scientometrics, 37, pp. 3-24.

CAMPANARIO, J.M. (1997). ¿Por qué a los científicos y a nuestros alumnos les cuesta tanto, a veces, cambiar sus ideas científicas? Didáctica de las Ciencias Experimentales y Sociales, 11, pp. 31-62.

CAMPANARIO, J.M. (1998a). ¿Quiénes son, qué piensan y qué saben los futuros maestros y profesores de ciencias? Una revisión de estudios recientes. Revista Interuniversitaria de Formación del Profesorado. (En prensa.)

CAMPANARIO, J.M. (1998b). Ventajas e inconvenientes de la historia de la ciencia como recurso en la enseñanza de las ciencias. Revista de Enseñanza de la Física. (En prensa.)

CARIN, A.A. y SAND, R.B. (1985). Teaching modern science. Columbus, Ohio: Merrill.
CARRASCOSA, J. y GIL, D. (1985). La «metodología de la superficialidad» y el aprendizaje de las ciencias. Enseñanza de las Ciencias, 3, pp. 113-120.

CARRETERO, M. (1987). A la búsqueda de la génesis del método científico: Un estudio sobre la capacidad de eliminar hipótesis. Infancia y Aprendizaje, 38, pp. 53-68.

CARRETERO, M. y BAILLO, M. (1995). Effects of counterevidence on conceptual change in Physics and History. Ponencia presentada en la V EARLI Conference. Aix en Provence, Francia.

CARRETERO, M. y LIMÓN, M. (1995). The theoretical basis of consctructivism and its implications for instructional design. Ponencia presentada en la V EARLI Conference. Aix en Provence, Francia.

CARTER, G.S. y SIMPSON, R.D. (1978). Science and reading: A basic duo. The Science Teacher, 45, p. 20.

CHALMERS, A.F. (1982). ¿Qué es esa cosa llamada ciencia? Madrid: Siglo XXI Editores.

CHAMPAGNE, A.B., KLOPFER, L.E. y GUSTONE, R.F. (1982). Cognitive research and the design of science instruction. Educational Psychologist, 17, pp. 31-53.

CLEMENT, J., BROWN, D.E. y ZIETSMAN, A. (1989). Not all preconceptions are misconceptions: finding «anchoring conceptions» for grounding instruction on students' intuitions. International Journal of Science Education, 11, pp. 554-565.

DREYFUS, A., JUNGWIRTH, E. y ELIOVITCH, R. (1990). Applying the «cognitive conflict» strategy for conceptual change - some implications, difficulties and problems. Science Education, 74, pp. 555-569.

DRIVER, R. (1988). Un enfoque constructivista para el desarrollo del currículo de ciencias. Enseñanza de las Ciencias, 6, pp. 109-120.

DUIT, R. (1991). Students' conceptual framework consequences for learning science, en Glynn, S., Yeany, R. y Britton, B. (eds.).The Psychology of Learning Science. Hillsdal: Lawrence Erlbaum.

ESLER, W.K. y ESLER, M.K. (1985). Teaching elementary science. Belmont: Wadsworth.

FULWILER, T. (1987).The Journal Book. Portsmouth: Boynton/ Cook.

FURIÓ, C. (1994). Tendencias actuales en la formación del profesorado de ciencias. Enseñanza de las Ciencias, 12, pp. 188-199.

GARCÍA, J.J. y CAÑAL, P. (1995). ¿Cómo enseñar? Hacia una definición de las estrategias de enseñanza por investigación. Investigación en la Escuela, 25, pp. 5-16.

GARCÍA-ARISTA, E., CAMPANARIO, J.M. y OTERO, J.C. (1996). Influence of subject matter setting on comprehension monitoring. European Journal of Psychology of Education, 11, pp. 427-441.

GIL, D. (1983). Tres paradigmas básicos en la enseñanza de las ciencias. Enseñanza de las Ciencias, 1, pp. 26-33.

GIL, D. (1987). Los programas-guía de actividades: Una concrección del modelo constructivista de aprendizaje de las ciencias. Investigación en la Escuela, 3, pp. 3-12. 
GIL, D. (1993). Contribución de la historia y de la filosofía de las ciencias al desarrollo de un modelo de enseñanza-aprendizaje como investigación. Enseñanza de las Ciencias, 11, pp. 197212.

GIL, D. (1994). Relaciones entre conocimiento escolar y conocimiento científico. Investigación en la Escuela, 23, pp. 17-32.

GIL, D., CARRASCOSA, J., FURIÓ, C. y MARTÍNEZTORREGROSA, J. (1991). La enseñanza de las ciencias en la educación secundaria. Barcelona: ICE. Universitat Autònoma de Barcelona.

GIL, D., MARTÍNEZ-TORREGROSA, J. y SENENT, F. (1988). El fracaso en la resolución de problemas de física: Una investigación orientada por nuevos supuestos. Enseñanza de las Ciencias, 6, pp. 131-146.

GIL, D. y VALDÉS, P. (1995). Contra la distinción clásica entre «teoría», «prácticas experimentales» y «resolución de problemas»: el estudio de las fuerzas elásticas como ejemplo ilustrativo. Didáctica de las Ciencias Experimentales y Sociales, 9, pp. 3-25.

GUNSTONE, R.F. y NORTHFIELD, J. (1994). Metacognition and learning to teach. International Journal of Science Education, 16, pp. 523-537.

GUNSTONE, R.F. y WHITE, R. (1981). Understanding of gravity. Science Education, 65, pp. 291-299.

HAMMER, D. (1994). Epistemological beliefs in introductory Physics. Cognition and Instruction, 12, pp. 151-183.

HEWSON, P.W. (1985). Epistemological commitments in the learning of science: Examples from dynamics. European Journal of Science Education, 7, pp. 163-172.

HEWSON, P.W. (1992). El cambio conceptual en la enseñanza de las ciencias y la formación de los profesores. Ponencia presentada en el encuentro sobre Investigación y Desarrollo del Currículo en la Enseñanza de las Ciencias. Madrid: CIDE.

HEWSON, P.W. y BEETH, M.E. (1995). Enseñanza para un cambio conceptual: Ejemplos de fuerza y movimiento. Enseñanza de las Ciencias, 13, pp. 25-35.

HEWSON, P. y HEWSON, M. (1984). The role of conceptual conflict in conceptual change and the design of science instruction. Instructional Science, 13, pp. 1-13.

HEWSON, P.W. y THORLEY, N.R. (1989). The conditions of conceptual change in the classroom. International Journal of Science Education, 11, pp. 541-553.

HODSON, D. (1994). Hacia un enfoque más crítico del trabajo de laboratorio. Enseñanza de las Ciencias, 12, pp. 299-313.

LENOX, R.S. (1985). Education for the serendipitous discovery. Journal of Chemical Education, 62, pp. 283-285.

LIGHTMAN, A. y GINGERICH, O. (1991). When do anomalies begin? Science, 255, pp. 690-695.

LINDER, C. (1993). A challenge to conceptual change. Science Education, 77, pp. 293-300.

LOMBARDI, O.I. (1997). La pertinencia de la historia en la enseñanza de las ciencias: argumentos y contraargumentos. Enseñanza de las Ciencias, 15, pp. 343-349.

LOPES, B. y COSTA, N. (1996). Modelo de enseñanza-aprendizaje centrado en la resolución de problemas: Fundamentación, presentación e implicaciones educativas. Enseñanza de las Ciencias, 14, pp. 45-61.

LLEDÓ, A.I. y CAÑAL, P. (1993). El diseño y desarrollo de materiales curriculares en un modelo investigativo. Investigación en la Escuela, 21, pp. 10-19.

MELLADO, V. y CARRACEDO, D. (1993). Contribuciones de la filosofía de la ciencia a la didáctica de las ciencias. Enseñanza de las Ciencias, 11, pp. 331-339.

MENESES, J.A. (1992). Un modelo didáctico con enfoque constructivista para la enseñanza de la física en el nivel universitario. Revista Interuniversitaria de Formación del Profesorado, 14, pp. 93-106.

NOVAK, J.D. (1982). Teoría y práctica de la educación. Madrid: Alianza Universidad.

NOVAK, J.D. y GOWIN, D.B. (1988). Aprendiendo a aprender. Barcelona: Martínez Roca.

NUSSBAUM, J. y NOVICK, S. (1982). Alternative frameworks, conceptual conflict and accomodation: towards a principled teaching strategy. Instructional Science, 11, pp. 183-200.

OSBORNE, J.F. (1996). Beyond constructivism. Science Education, 80, pp. 53-82.

OSBORNE, R. y FREYBERG, P. (1985). Learning Science. Auckland: Heineman.

OTERO, J.C. (1990). Variables cognitivas y metacognitivas en la comprensión de textos científicos: el papel de los esquemas y el control de la propia comprensión. Enseñanza de las Ciencias, 8, pp. 17-22.

OTERO, J.C. y CAMPANARIO, J.M. (1990). Comprehension evaluation and regulation in learning from science texts. Journal of Research in Science Teaching, 27, pp. 447-460.

OTERO, J.C., CAMPANARIO, J.M. y HOPKINS, K.D. (1992). The relationship between academic achievement and metacognitive comprehension monitoring ability of Spanish secondary school students. Educational and Psychological Measurement, 52, pp. 419-430.

PINTÓ, R., ALIBERAS, J. y GÓMEZ, R. (1996). Tres enfoques de la investigación sobre concepciones alternativas.Enseñanza de las Ciencias, 14, pp. 221-232.

POSNER, G.J., STRIKE, K.A., HEWSON, P.W. y GERTZOG, W.A. (1982). Accomodation of a scientific conception: Toward a theory of conceptual change. Science Education, 66, pp. 211-227.

POZO, J.I. (1987). La historia se repite: Las concepciones espontáneas sobre el movimiento y la gravedad. Infancia y Aprendizaje, 38, pp. 69-87.

POZO, J.I. y CARRETERO, M. (1987). Del pensamiento formal a las concepciones espontáneas: ¿Qué cambia en la enseñanza de la ciencia? Infancia y Aprendizaje, 38, pp. 35-52.

POZO, J.I., SANZ, A., GÓMEZ, M.A. y LIMÓN, M. (1991). Las ideas de los alumnos sobre la ciencia: Una interpretación desde la psicología cognitiva. Enseñanza de las Ciencias, 9, pp. 83-94.

RAGHAVAN, K. y GLASER, R. (1995). Model-based analysis and reasoning in science: The MARS curriculum. Science Education, 79, pp. 37-61. 
RESNICK, L.B. (1983). Toward a cognitive theory of instruction, en Paris, S., Olson, G. y Stevenson, H. (eds.). Learning and motivation in the classroom. Hillsdale, Nueva Jersey: Lawrence Erlbaum.

ROWELL, J.A. y DAWSON, C.J. (1983). Laboratory counterexamples and the growth of understanding in science.European Journal of Science Education, 4, pp. 299-309.

SÁNCHEZ, G. yVALCÁRCEL, M.V. (1993). Diseño de unidades didácticas en el área de ciencias experimentales. Enseñanza de las Ciencias, 11, pp. 33-44.

SCHMIDT, K.G. (1995). Problem-based learning: An introduction. Instructional Science, 22, pp. 247-250.

SEGURA, D. (1991). Una premisa para el cambio conceptual: El cambio metodológico. Enseñanza de las Ciencias, 9, pp. 175-180.

SMITH, E.L., BLAKESLEE, T.D. y ANDERSON, C.W (1993). Teaching strategies associated with conceptual change learning in science. Journal of Research in Science Teaching, 30, pp. 111-126.
STRIKE, K.A. y POSNER, G.J. (1990). A revisionist theory of conceptual change, en Duschl, R. y Hamilton, R. (eds.). Philosophy of Science, Cognitive Science and Educational Theory and Practice. Albany, Nueva York: SUNY Press.

THIBERGHIEN, A., PSILLOS, D. y KOUMARAS, P. (1995). Physics instruction from epistemological and didactical bases. Instructional Science, 22, pp. 423-444.

TOBIN, K. y FRASER, B.J. (1990). What does it mean to be an exemplary science teacher? Journal of Research in Science Teaching, 27, pp. 3-25.

VAN ANDEL, P. (1994). Anatomy of the unsought finding. Serendipity: Origin, history, domains, traditions, appearances, patterns and programmability. British Journal of Philosophy of Science, 45, pp. 631-648.

VILLANI, A. y ORQUIZA, L. (1995). Conflictos cognitivos, experimentos cualitativos y actividades didácticas. Enseñanza de las Ciencias, 13, pp. 279-294.

WEST, L.H.T. y PINES, A.L. (1983). How «rational» is rationality? Science Education, 67, pp. 37-39.

[Artículo recibido en junio de 1997 y aceptado en marzo de 1998.] 